\title{
Vulnerabilities of information technologies
} Impact on the Information Society

\author{
Klaus Brunnstein \\ Professor for Application of Informactics, Faculty for Informatics, University of Hamburg, \\ Germany, Tel: +49-40-42883-2406; Fax: +49-40-42883-2226
}

brunnstein@informatik.uni-hamburg.de; http://agn-www.informatik.uni-hamburg.de

Abstract: Faster than any technology before, Information and Communication technologies (ICTs) continue to change economies and societies in ways affecting many aspects of human life. Already after a short time organisations and individuals have become so dependent upon proper functioning of highly complex and hardly understandable ICT-systems that any deviation from "normal" behaviour may have adversary, if not damaging effects. Contemporary technologies are in many cases designed and implemented without adequate provisions for safe and secure functioning. These systems can easily be attacked, even by experimenting boys at the age of puberty. e.g. by injecting viruses and worms into The Internet, which then rapidly propagates these malicious gifts (in some cases even in epidemic amounts of up to 100 million emailed worms per 24 hours) into enterprises, offices, schools and everybody's PC. Internet communication protocols are weakly designed, and it is easy to "spoof" ones email address, to "sniff" and to intercept messages, such as transfer of electronic funds. To protect these already overly complex systems, the usual solution is to add more complexity: firewalls, antivirus software and encryption. Two types of reaction to ICT related vulnerabilities can be observed. Some users have a "don't care" strategy, especially young people who leave a data trail of personal behaviour when surfing websites with potentially interesting economic or sociological content. Other users wish to exclude all risks and follow a strategy of "don't use". Both reactions are undesirable in the Information Society. Education on how to work with unsafe and insecure systems may help to protect users from undesired side-effects of ICT work.

Key words: computer crime, malicious software, risk analysis, risk management, safety, security, theory of economic cycles, vulnerabilities 


\section{INTRODUCTION: FROM INDUSTRIAL TO INFORMATION SOCIETIES}

In the history of mankind, several technologies have contributed to change economic and individual conditions and perspectives. Most technical inventions - such as horse-driven cars, windmills and ships - developed rather slowly, both the technology and the impact on society. However, James Watt's invention (1761) of the vapour-pressure driven "machine" changed economic conditions and in their wake the world order at significantly higher pace. In a first - stationary - form heavy machines with relatively small power (though large compared to human "manufacturing") produced industrial goods in what was to be the start of essential change in the production of goods. This change gained speed and impact when Watt's machines became sufficiently light and powerful to drive wagons on iron rails ("locomotion"). This allowed faster transportation, both of material resources to the fabrication sites and of products to consumers. The "Industrial Society" even gained more momentum with the application of other technical inventions such as electric energy, telephone and energy production from oil and uranium.

In 1960, when computers began to significantly spread into (then large) businesses and universities, the so called (technically) "developed countries" showed significant economic and societal changes since the advent of Watt's engine. These changes affected many areas: from hierarchical to democratic organisations, legal systems to support individual rights, opportunities for education and medical support for all, etc. But seen over the span of about 200 years of industrial history (1762-1960), the speed of change was rather slow.

Table 1. Technologies supporting development cycles of Industrial Societies

\begin{tabular}{lll}
\hline Cycle 1 & $(1760+)$ & Vapour driven stationary \\
& & machine (James Watt: 1762) \\
Cycle 2 & $(1810+)$ & Vapour-driven mobile \\
& & machine \\
Cycle 3 & $(1860+)$ & Oil-driven engines \\
Cycle 4 & $(1910+)$ & Electricity-driven engines, \\
& & networks \\
\hline
\end{tabular}

Analysis of similarities and differences in development of industrial technologies may help to predict future developments of ICTs. Schumpeter, Kondratieff et al. have observed cyclic behaviours in industrial economies when new technologies stimulated economic growth. Looking at essential 
("lead") technologies industrial developments can be classified into essentially 4 cycles, each with about $40-50$ years of duration ( $\sim 45$ years). See Table 1.

Faster than industrial technologies before, Information and Communication technologies (ICTs) continue to change economies and societies in ways affecting many aspects of human lives. ICTs are lead technologies of the Information Societies (Table 2.). Cycle 1 of Information Societies depended on initially large, heavy and difficult to handle "engines" (mainframes and their software) operated by specialists in "computer centres" - similar to factories in the Industrial economies - which developed into smaller and more powerful machines (Mini, Micro and Personal Computers). This made it important to transport digital results between remote sites and networks developed both within enterprises (Intranets, Local Area Networks) and globally. "Traditional" communication - telephony using terrestrial cables, satellite and mobile cell-based communication - has enlarged human communication space even in its previously analogous, but now digitalized forms. Cables are still the major basis of digital networks, but in some areas flexible mobile communication is a major factor of information exchange in enterprises and daily life. The development of The Internet - designed and intended for scientific and military communication - became the major "lead" technology for the 2 nd Cycle, similar to the development of railway transport in the 2nd Cycle of the Industrial Age.

Table 2. Technologies supporting development cycles of Information Societies

\begin{tabular}{|c|c|c|}
\hline Cycle 1 & $(1940+)$ & $\begin{array}{l}\text { Computer: Mainframe .. PC .. Chips; } \\
\text { stationary, local code/control; } \\
\text { computer companies support economic development. }\end{array}$ \\
\hline Cycle 2 & $(1985+)$ & $\begin{array}{l}\text { LAN ... WAN, mobile code/agents; } \\
\text { data searching \& mining, value-added services; } \\
\text { network companies lead development. }\end{array}$ \\
\hline Cycle 3 & $(2030+)$ & $\begin{array}{l}\text { ??? (Nano-miniaturisation: Quantum/Optical Computing) } \\
\text { ??? }\end{array}$ \\
\hline
\end{tabular}

In the Industrial age, railways enabled faster transport of resources, products and persons changing economic and human relationships as these progressed from local to regional, and finally to global activities. In similar ways Information and Communication technologies have started to rapidly and deeply affect many relationships that originate from Industrial societies. 


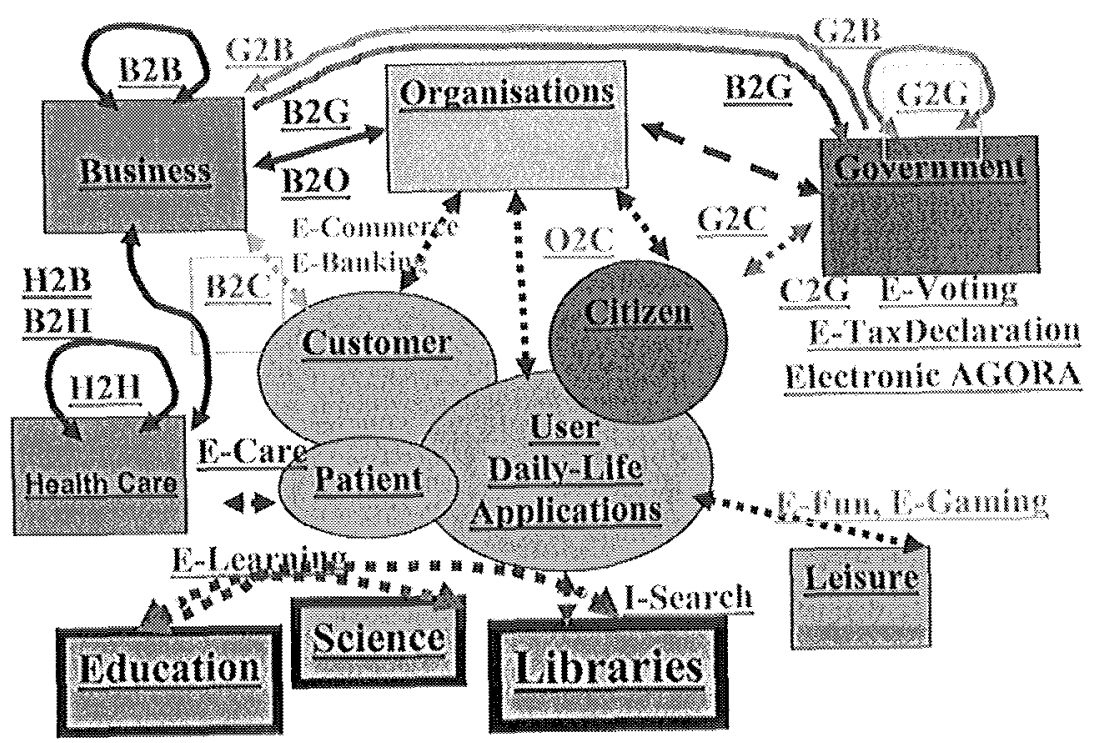

Figure 1. Relations in e-Society

Digital communication is facilitated by Local-Area Networks (LANs) and the Internet as a cooperation of many Wide-Area Networks (WANs). Based on the uniform Internet communication Protocol (IP) it is changing many relationships (see Figure 1.) in and between enterprises (Business to Employee, B2E; Business to Business, B2B), but also between business and customers (business to customer, B2C) and governments (B2G). Also, though with lower speed, individual relationships are changing: for patients (Health to Patient, H2P), for students (School to Student, S2S; Student to University, S2U) and citizen (Citizen to Government, C2G).. Essential processes of the Information and Communication Societies will be based upon production of digital values such as Information Search (Data Mining, I-Search). For example, changes in education such as distant and e-Learning, as well as in democratic processes, e.g. e-voting, are underway.

Remark: following contemporary implementation, such processes are labeled "e"for "electronic", thus indicating that in the implementation electromagnetic media and processes are used. As future technologies will possibly also other technologies such as photonic and quantum technologies, it seems advisable to label such relations as "d-relations" thus reflecting their digital (rather than analog) nature. 
It is true that speed and impact of developments in ICTs vary greatly over the planet. As in the Industrial Age, several countries (better: regions within nations) are advancing rapidly in technical developments with associated benefits, especially transfer of related products and methods to other parts of the world. The uneven distribution of regional development also implies that e-relationships develop rather differently over the planet. The "digital gap" hinders less "developed areas" to participate in this global process. On the other side, this "digital gap" protects less-developed areas from unwished side-effects and serious (e.g. security) problems of these technologies which affect enterprises, governments, organisations and individuals with undesired effects.

\section{VULNERABILITIES OF INFORMATION AND COMMUNICATION TECHNOLOGIES}

Already after such a short time organisations and individuals have become dependant upon proper functioning of these highly complex and hardly understandable systems. Any deviation from "normal" behaviour may have adversary, if not damaging, effects. Daily experience of "users" of Information and Communication Technologies is that computerized systems fail, rather often for reasons that a "normal user" can hardly analyse or understand. Failures range from unforeseen crashes, infections with malicious software (in many cases imported by Intranet or Internet communication) to loss of data and programs, to complete loss of function and connectivity.

A Risk Analysis of contemporary ICTs reveals a variety of reasons why such systems fail. Following the "life cycle" of ICT concepts and products, risks can be graded into "vulnerability classes":

- Paradigmatic risks: paradigms dominating design;

- Implementation risks: deficiencies in the quality of production and products

- Usage risks: risks in the ways systems are used

- Criminal risks: in addition, ICTs may be deliberately misused for criminal purpose.

\section{Paradigmatic risks (Vulnerability Class 1)}

Risks in this class are embedded in assumptions made in the design process and in the methods applied in production (implementation) of 
hardware, systems and application software. Take for example the concept that complex problems can be solved by cutting them into parts ("modules") which can separately be produced, to be subsequently combined in systems of high complexity. Not only are there the problems of adequate "cutting" of modules and of designing adequate interoperation, but contemporary systems also have become so complex that even experts can hardly understand their effects. To get still more functionality and interoperability, complex systems are combined to produce even higher levels of complexity. When systems from different origin with no common "interface" are combined, instruments are needed to "glue" such systems together; such "glueware" - "script" programming languages such as Java or Virtual BASIC - must be powerful to be able to support many different adaptations, but then also can be easily manipulated even by less qualified "script kiddies". The plenitude of malicious software (computer viruses, network worms, Trojan horses, trapdoors, backdoors, spyware, etc) is essentially based on script languages used for example. in office systems (Visual Basic for Applications etc.). In summary, concepts and tools used in the design process very deeply influence both the functions and the risks of digital technologies.

\section{Risks from inadequate implementation (Vulnerability Class 2)}

The production of digital technologies, especially system and application software (and to a lesser degree also of hardware and their "drivers"), has many weaknesses. The most evident are: inadequate qualification of programmers, and inadequate testing and production under heavy time pressure. Conceptual and programming errors have effects, mostly for users, when software behaves unpredictably, including destruction of achieved work and broken connections. The number of experienced "computer emergencies" is rapidly growing, with sometimes millions of servers and even more local computers being affected by software weaknesses and "infections" by network "worms".

Effects of software weaknesses of course materialise predominantly on systems of the most dominant system and software producers. Microsoft therefore globally leads in sold software and in software flaws, but is also in the lead with malicious software living on Microsoft's insecure software design and implementation. Table 3. shows a list of programming faults, detected within last year. Although there are also many problems with nonMicrosoft systems (e.g. Linuxes), Microsoft dominates in those incidents where in some cases many millions computer systems were affected. 
Table 2. Vulnerabilities reported by CERT/CC (CERT/CC October 2002-October 2003)

\begin{tabular}{ll}
\hline A1 & Buffer Overflow Vulnerability in Core Windows DLL \\
A2 & Remote Buffer Overflow in Sendmail \\
A3 & Increased Activity Targeting Windows Shares \\
A4 & Samba Contains Buffer Overflow in SMB/CIFS Packet Fragment Reassembly Code \\
A5 & MS-SQL Server Worm \\
A6 & Multiple Vulnerabilities in Implementations of the Session Initiation Protocol (SIP) \\
A7 & Multiple Vulnerabilities in SSH Implementations \\
A8 & Buffer Overflow in Microsoft Windows Shell \\
A9 & Double-Free Bug in CVS Server \\
A10 & Buffer Overflow in Windows Locator Service \\
& \\
B1 & Integer overflow in Sun RPC XDR library routines \\
B2 & Multiple Vulnerabilities in Lotus Notes and Domino \\
B3 & Buffer Overflow in Sendmail \\
B4 & Multiple Vulnerabilities in Snort Pre-processors \\
& \\
C1 & Multiple Vulnerabilities in Snort Pre-processors \\
C2 & Exploitation of Vulnerabilities in Microsoft RPC Interface \\
C2.a & W32/Blaster Worm \\
C2.b & W32/Welchia \\
C3 & Cisco IOS Interface Blocked by IPv4 Packet \\
C4 & Vulnerabilities in Microsoft Windows Libraries \& Internet Explorer \\
C4.a & Buffer Overflow in Microsoft Windows HTML Conversion Library \\
C4.b & Integer Overflows in Microsoft Windows DirectX MIDI Library \\
C4.c & Multiple Vulnerabilities in Microsoft Internet Explorer \\
C5 & Malicious Code Propagation and Antivirus Software Updates \\
& \\
D1 & W32/Mimail Variants (added: plus Paylap variants) \\
D2 & Buffer Overflow in Windows Workstation Service \\
D3 & Multiple Vulnerabilities in Microsoft Windows and Exchange \\
D4 & Multiple Vulnerabilities in SSL/TLS Implementations \\
D5 & Exploitation of Internet Explorer Vulnerability \\
D6 & W32/Swen.A Worm \\
D7 & Buffer Overflow in Sendmail \\
D8 & Buffer Management Vulnerability in OpenSSH \\
D9 & RPCSS Vulnerabilities in Microsoft Windows \\
\hline
\end{tabular}

\section{Risks from usage (Vulnerability Class 3)}

Not surprisingly, usage of unsafely designed and insecurely implemented software presents additional risks. When installation and administration of system and application software is improperly performed at user sites, this may adversely affect performance and proper functioning. Due to the 
complexity, as well as inadequate documentation, users hardly understand the effects of their attempts to "properly" use such systems. Consequently users apply "trial and error" methods in learning to work with new features, rather than trying methodologically to understand which functions may have which effects, and which precautions should be taken to avoid unwished side-effects. This somewhat "explorative" way of using a system rather often leads to a risky attitude, with potentially hazardous effects. For example clicking on unknown attachments without due care.

Software manufacturers often argue that failure of software is mainly caused by improper actions of users. But in many - if not most - cases, the human-computer interface (e.g. the display of functions and operations on the screen, or the handling of input devices such as mouse and keyboard) is inadequately designed. Users are not properly supported by help functions, which, when existing, in many cases are so complex that users are further mislead. Because users have a primary interest in doing their work, they rather often tend to forget precautions and in some cases even bypass security measures when they think that their work performance is reduced.

\section{Risks from deliberate misuse (Vulnerability Class 4)}

Digital Information and Communication technologies provide many opportunities for deliberate misuse, including criminal misuse. Only few cases of criminal misuse have been reported and prosecuted. Some of these were broadly covered in the media although few caused major damage (such as the SoBig worm affecting some 100 millions of emails and several 10.000 enterprise servers). Deliberate misuse for criminal purposes has not yet significantly impacted on business and government. Consequently, both legal provisions and prosecution capabilities are less developed than in other areas of criminal law. Still, there can be no doubt that further development of ICTs will be associated with growing misuse considering that Class 1 and Class 2 vulnerabilities are so dominant in contemporary ICTs.

\section{IMPACT OF VULNERABILITIES ON INFORMATION AND KNOWLEDGE SOCIETIES}

Just as industrial technologies in the industrial age, Information and Communication Technologies will unavoidably affect many (though not all) parts of human organisations, economies, government and individual lives. As in the industrial society, ICTs are driven by supply-side concepts, with 
hardly any analysis of customer impact made by ICT developers. Consequently, such impacts overcome users - who seldom have the choice of avoiding ICT applications - without any possibility to understand or contain undesirable effects.

Some of this materialised in the first cycle of the Information Society (stationary operation) with complex systems where nobody can assure correctness of results in any detail. Blind reliance has developed among users: "this must be true because this was produced by a computer". Overreliance and risk acceptance are even now, at the beginning of the 2 nd cycle (network-based operation), still dominant. Just as lemmings, animals who blindly follow their forerunners, users tend to accept risks of PCs and The Internet which they feel are unavoidable. In some sense, the more technologically advanced a society is, the more risks are blindly accepted. Sociologists and philosophers observe this to be a general pattern in contemporary societies, label these as "risk societies" (Beck 1999).

Also impact on individual attitude and behaviour can also be observed. While email, as tool of direct and fast communication, supports personal and business needs very well, it also forces users to adapt. At best email forces users to react quickly and thus tends to dominate time management of users: if you expect a fast reaction, you must also react quickly, independent of whatever else you are doing. Replying to email overshadows any other priority. This is especially a nuisance when unwished email requires reaction, such as malicious software requesting sanitary actions or floods of undesired email (spamming).

In general, time management changes significantly when using contemporary communication systems. Similar to workers in industrial factories, users of interconnected ICT-systems behave as slaves of engines which they can hardly understand and control. It remains a major task of education in the Information Age to enable users to master these engines rather than becoming controlled by them.

\section{ACTIONS FOR REDUCING RISKS OF CONTEMPORARY ICTS}

In general, risks may be reduced in several ways:

- "Risk avoidance": Information and Knowledge systems must be structured in such a way that a class of given risks cannot materialise; for example, this strategy implies that a system is designed and constructed so that it cannot fail.

- "Risk reduction": methods and mechanisms must be realised which reduce undesired effects when some risk materialises, hopefully with 
lower probability; for example, this strategy implies that a system may fail, but that there are curative mechanisms which reduce the damage when the system fails.

- "Risk acceptance": nothing preventive or curative is done ("don't care" strategy); for example, although one knows that the system is vulnerable, one simply hopes that nothing will happen and therefore does nothing to prevent or reduce the risk.

While risk acceptance is what the vast majority of users practice, risk reduction is the strategy which many enterprises and governments presently apply. To reduce the impact of crashing systems and programs, "computer viruses" and "worms", hacker attacks, mass distribution of undesired email, etc. special forms of security software (antivirus software, firewalls, intrusion detection systems, etc.) are deployed to reduce threats.

Risk acceptance may be regarded as an acceptable strategy as long as large damages can be avoided. This strategy will be no longer acceptable, when large damages materialise because of ineffective protection methods. By the end of the 2nd cycle of the Information Society, interdependence of systems will have reached a degree that many small failures will combine to blackouts similar to (though more serious than) recent power outages in the USA and Europe.

To avoid that networks become so strongly interconnected that any failure becomes "critical", the only solution available in the next decennium is to redesign basic technologies as to become inherently safe and secure. Regrettably mankind will only learn - as in the Industrial Age - from severe accidents, however urgent even today the need for safe and secure system designs.

\section{BIOGRAPHY}

Klaus Brunnstein is co-founder of the faculty for Informatics at Hamburg University and since 1973 professor for Application of Informatics. His special interests include: Data Protection, Computer and Network Security, Incident and Risk Analysis of IT Systems, Legal and Ethical Aspects of Informatics; Computers, Culture and Media. In 1988 he founded a Virus Test Centre (VTC) and in 1989 he started the first (2 year) course on IT Security and Incident Analysis. He is active in IFIP: the "International Federation for Information Processing" as German representative in IFIP Technical Committee-9 "Relationship between Computer and Society", as founding chairman of IFIP Working Group 9.2 "Social Accountability", and as elected IFIP President for two terms: 2002-2004 and 2004-2007. He has 
organised the programmes of several international conferences, among which are:

- 1989: ORAIS'89: Opportunities and Risks of Artificial Intelligence Systems, IFIP-GI Conference, Hamburg, 1989;

- 1998: Intellectual Property Rights KnowRight 2, IFIP World Computer Congress 1998, Vienna-Budapest, 1998;

- 2000: Intellectual Property Rights:KnowRight 3 \& InfoEthics, Wien, September 2000;

- 2002: Human Choice and Computers, IFIP World Computer Congress2002, Montreal;

- 2003: World IT Forum (WITFOR) August 2003, Vilnius/Lithuania.

\section{REFERENCES}

Beck, Ulrich (1999) World Risk Society, Polity Press, Malden.

Brunnstein, Klaus (1989-2003) Introduction into IT Security and Safety: lectures (1989-2003) esp. addressing Incident Handling, Risk Analysis and Risk Management, Forensic Informatics.

CERT/CC (October 2002-October 2003) Summaries CS-2003: March 21, 2003 (A), June 3, 2003 (B), September 8, 2003 (C), November 24, 2003 (D): Source: http://www.cert.org/current/current_activity.html.

Kondratieff, Nikolai D. (1935) "The Long Waves in Economic Life," Review of Economic Statistics 17(6) November 1935.

Kondratiev, Nikolai D. (1984) The Long Wave Cycle. Richardson \& Snyder, New York. 